\title{
The Role of Intragranular Nanopores in Capacity Fade of Nickel-Rich Layered Li( $\left(\mathrm{Ni}_{1-\mathrm{x}-\mathrm{y}} \mathrm{Co}_{\mathrm{x}} \mathrm{Mn}_{\mathrm{y}}\right) \mathrm{O}_{2}$ Cathode Materials
}

Shamail Ahmed, ${ }^{1}$ Anuj Pokle, ${ }^{1}$ Simon Schweidler, ${ }^{2}$ Andreas Beyer, ${ }^{1}$ Matteo Bianchini, ${ }^{2}$ Felix Walther, ${ }^{3}$ Andrey Mazilkin, ${ }^{2,4}$ Pascal Hartmann, ${ }^{2,5}$ Torsten Brezesinski, ${ }^{2}$ Jürgen Janek, ${ }^{2,3^{*}}$ and Kerstin Volz ${ }^{1^{*}}$

${ }^{1}$ Materials Science Centre and Faculty of Physics, Philipps University Marburg, Hans-Meerwein-Straße 6, 35043 Marburg, Germany

2 Battery and Electrochemistry Laboratory, Institute of Nanotechnology, Karlsruhe Institute of Technology (KIT), Hermann-von-Helmholtz-Platz 1, 76344 Eggenstein-Leopoldshafen, Germany

${ }^{3}$ Institute of Physical Chemistry \& Center for Materials Research, Justus-Liebig-University, Heinrich-BuffRing 17, 35392 Giessen, Germany

${ }^{4}$ Karlsruhe Nano Micro Facility, Karlsruhe Institute of Technology (KIT), Hermann-von-Helmholtz-Platz 1, 76344 Eggenstein-Leopoldshafen, Germany

${ }^{5}$ BASF SE, Carl-Bosch-Strasse 38, 67056 Ludwigshafen, Germany

Email: kerstin.volz@physik.uni-marburg.de 


\section{Secondary Particles}
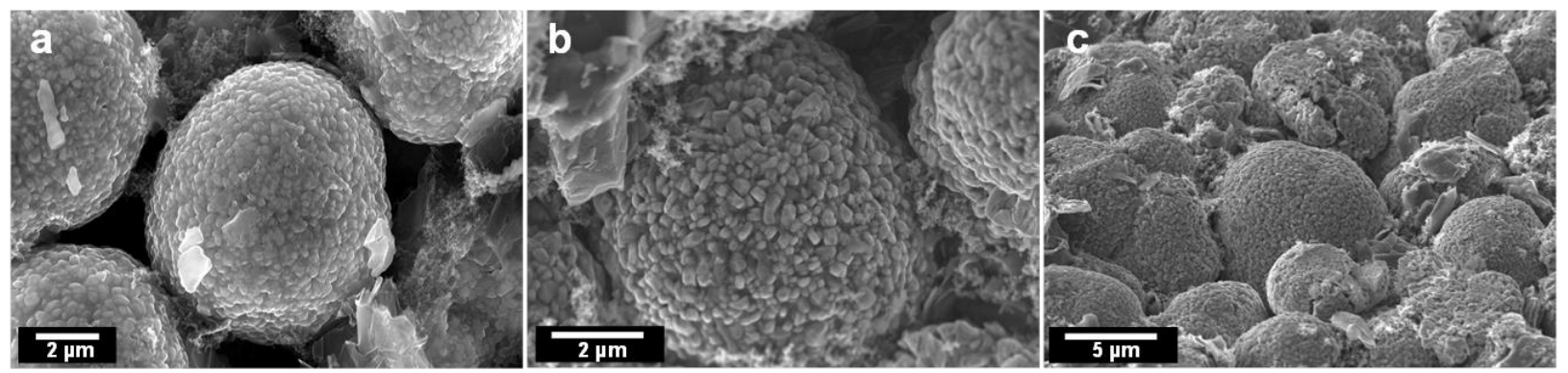

Figure S1. SEM micrographs showing secondary NCM851005 particles (a) in the pristine state and (b) after 200 and (c) 500 cycles.

\section{Metal Segregation and Rim Thickness}
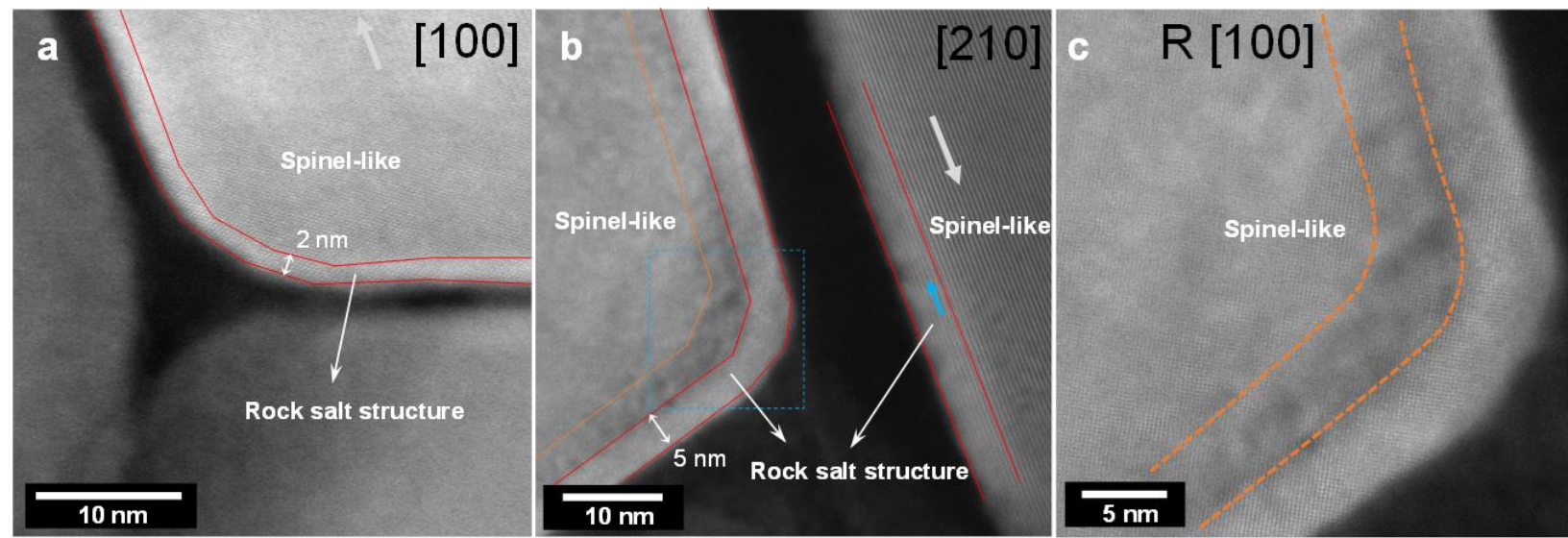

Figure S2. Rim thickness at the surface of primary particles from (a) pristine material, (b) and (c) after 500 cycles. Rims are marked with red lines. The orange lines in the figures (b) and (c) show pit regions. The rim thickness increases from $2 \mathrm{~nm}$ to $5 \mathrm{~nm}$ with cycling as indicated in (a) and (b). 


\section{Nanopore Evolution}

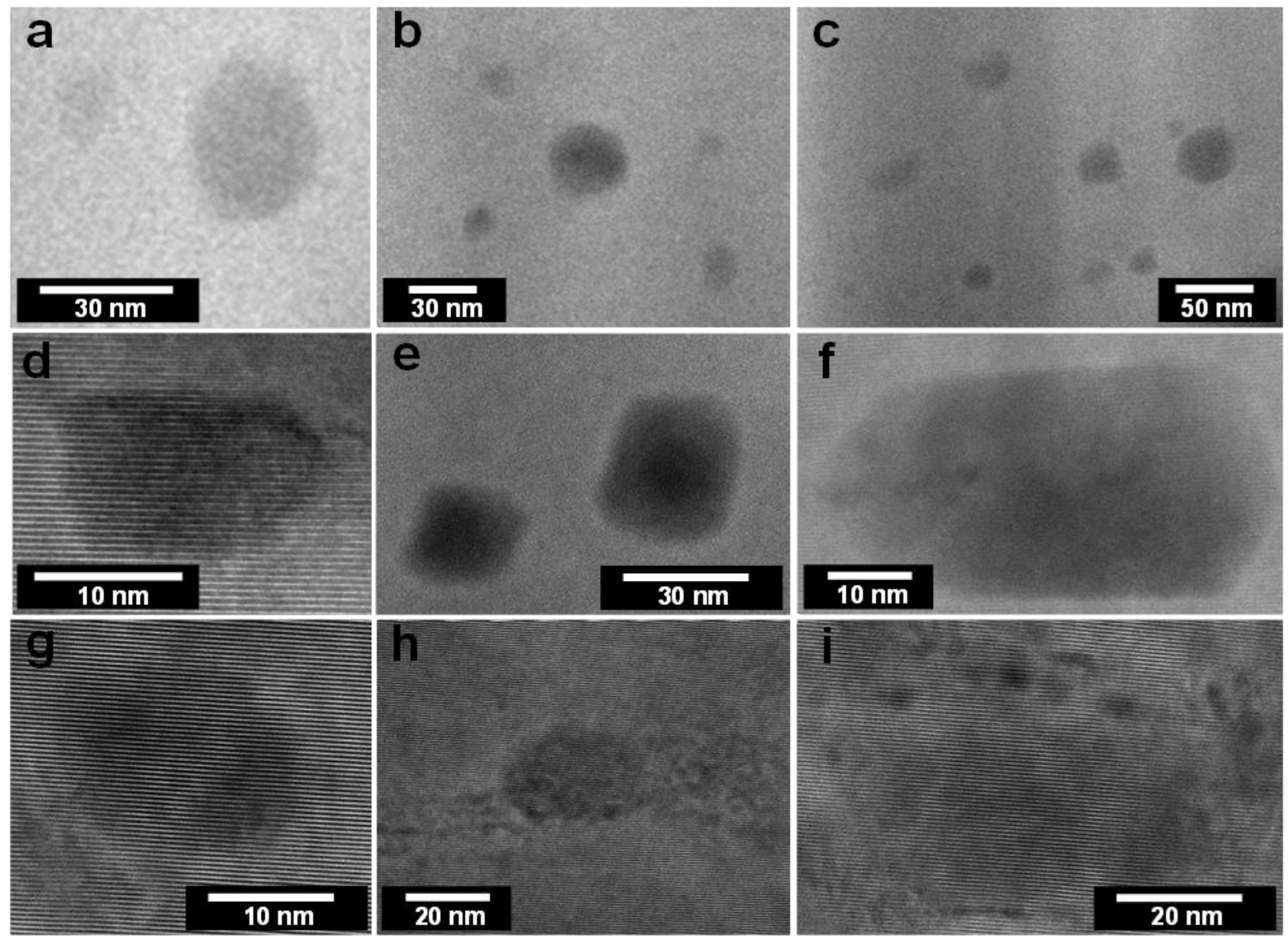

Figure S3. (a-c) Different regions of pristine NCM851005 showing nanopores of spherical shape. (d-f) Pores after 200 cycles showing a rectangular shape with sharp boundaries due to metal segregation. (g-i) Pores after 500 cycles showing various shapes and diffuse boundaries. 
High-Resolution Images from the Bulk and Nanopore Regions in the Samples after cycling
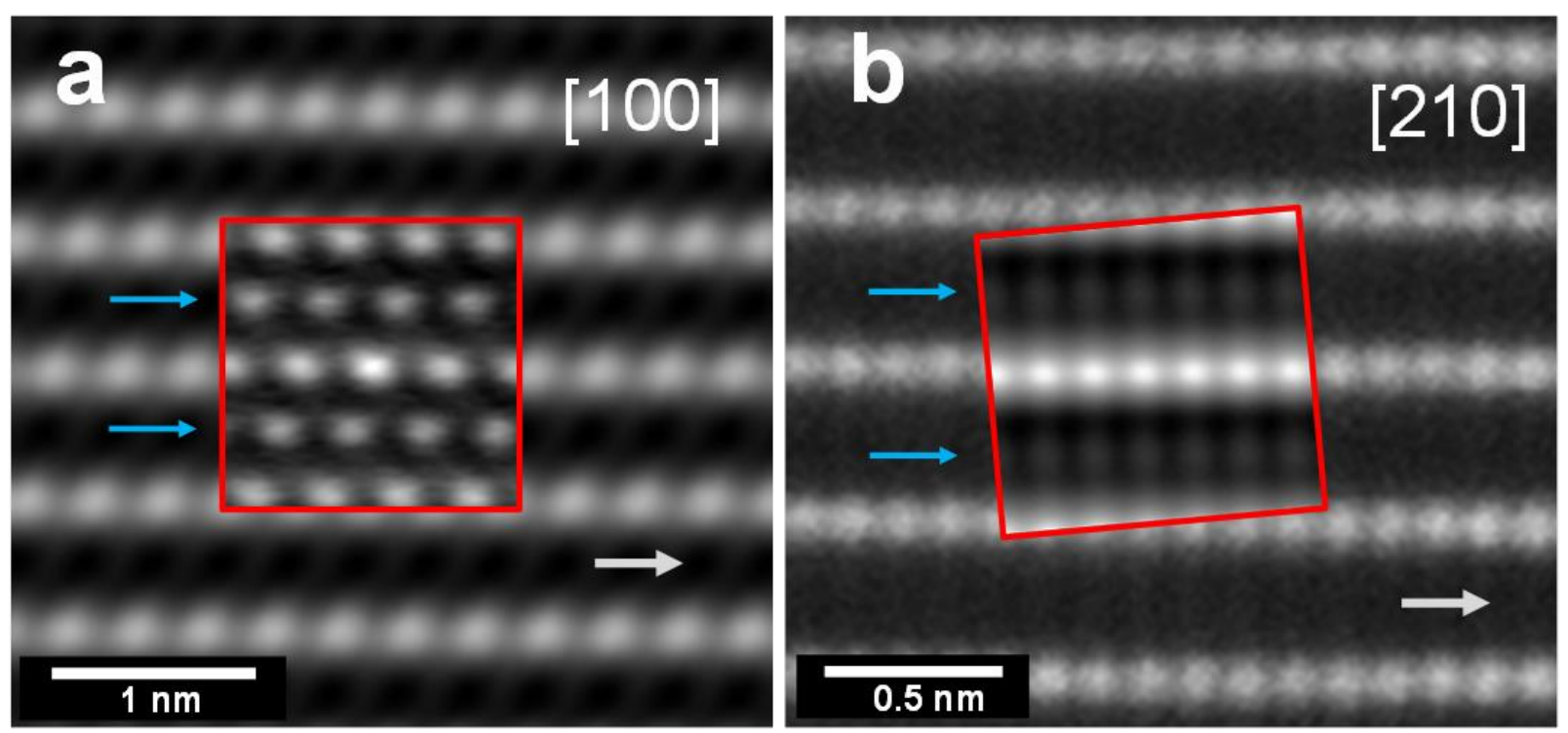

Figure S4.1. (a) and (b) are STEM-high-resolution images from regions far away from nanopores, from samples after 200 and 500 cycles, respectively, proving that away from the pores the layered structure can still be found. The insets in (a) and (b), marked by red squares, are average images of figures $3 d$ and $3 f$ respectively. The averaging procedure is described elsewhere. ${ }^{1}$ The intensity from the Li positions in the HAADF image indicates that the $\mathrm{Li}$ has been replaced by $\mathrm{Ni}$ and rock-salt structure has formed around nanopores, which is in projection. The grey arrows mark the direction parallel to the (003) plane, the blue arrows mark the positions of the Li positions. 

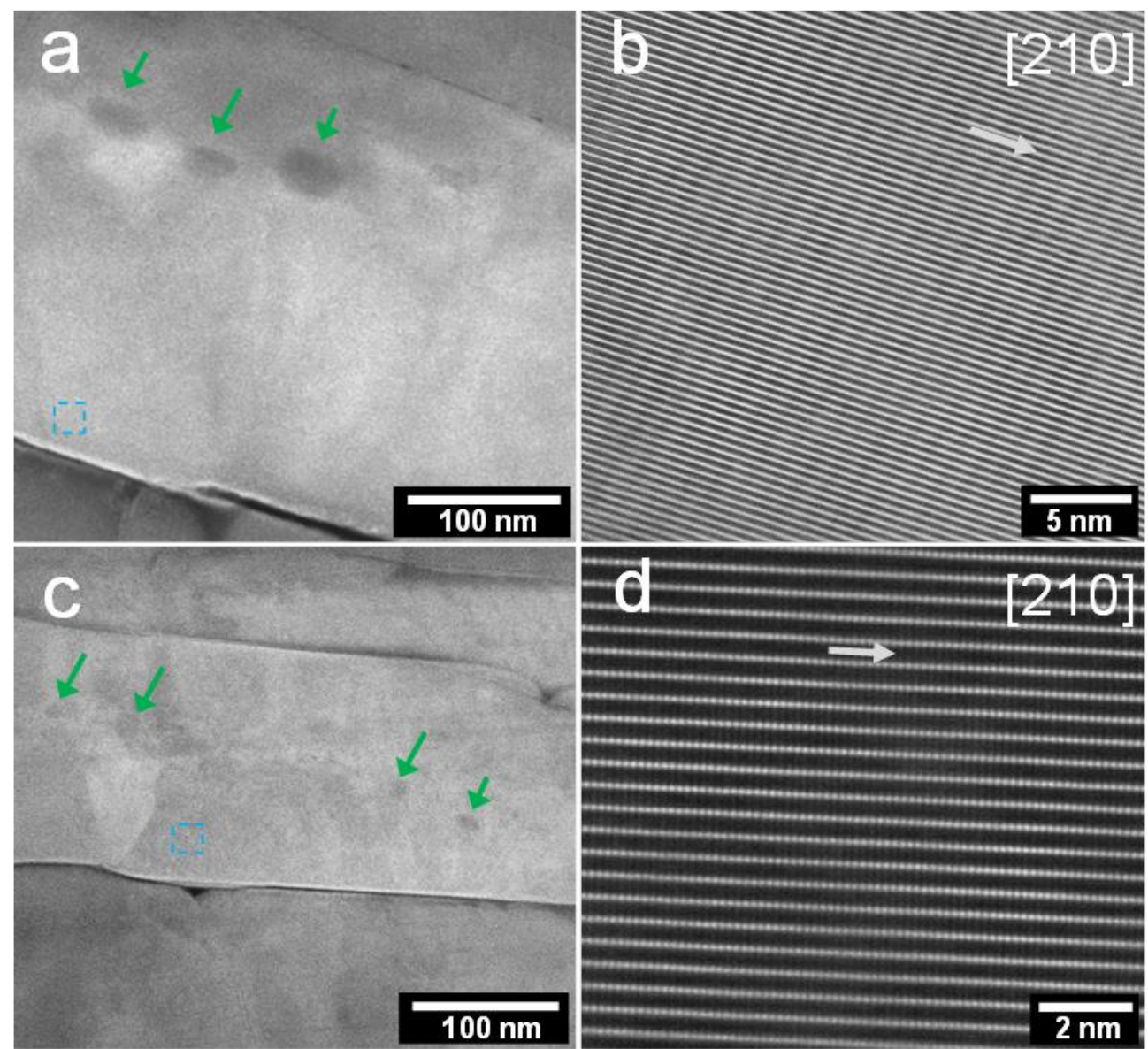

Figure S4.2. a) Low magnification STEM-HAADF image showing intragranular nanopores in a sample after 200 cycles. (b) High-resolution STEM-HAADF image of the region marked by dashed blue-bordered square in (a) showing layered structure. (c) Low magnification STEM-HAADF image showing intragranular nanopores in a sample after 500 cycles. (d) High-resolution STEM-HAADF image of the region marked by blue-bordered square in (c) showing layered structure. 
Model: Comparing Different Phases and Zone Axis
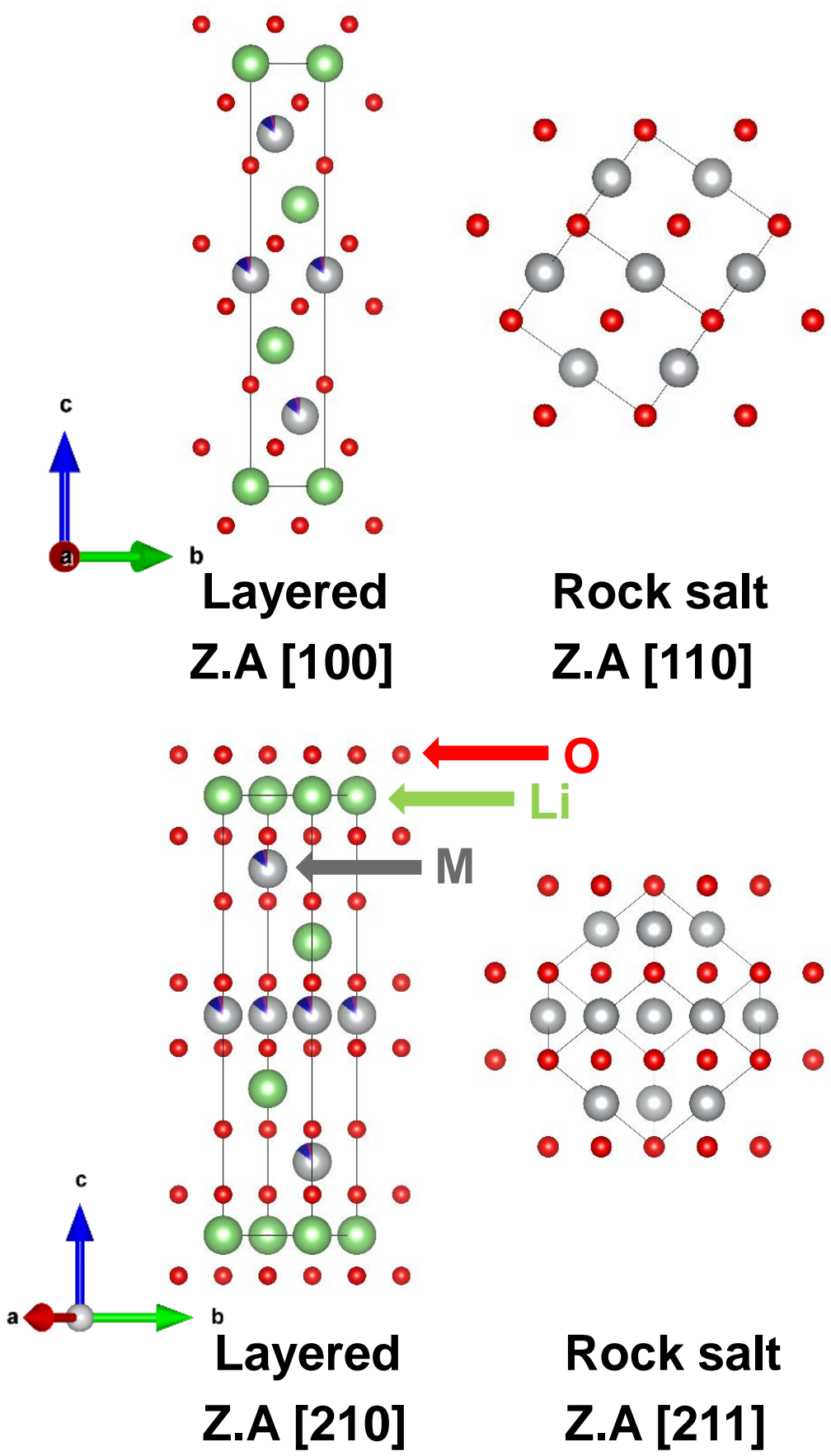

Figure S5. Comparison of two layered zone axes (in space group $R-3 m$ ), namely, [100] and [210] with their rock-salt phase counterparts (in space group $F m-3 m$ ), [110] and [211], respectively. The models correspond to Figures $3 \mathrm{~d}$ and $\mathrm{f}$, respectively, when viewed in the respective directions. 


\section{EELS - Electron Energy Loss Spectroscopy}

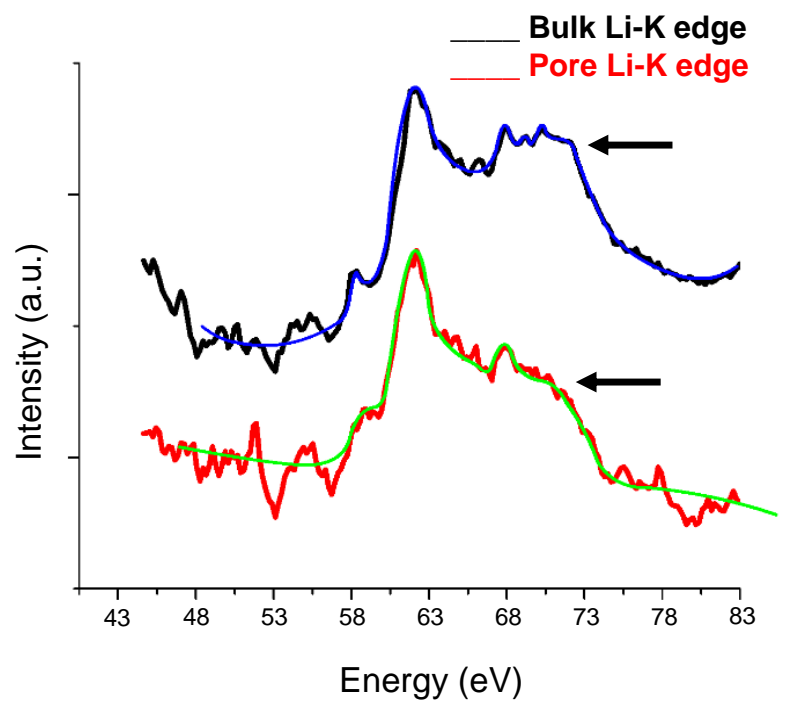

Figure S6. EELS spectra of the Li-K edge from the bulk and nanopore regions. The smoothened peak fits (blue and green) are superimposed on the background-corrected data. The spectra are offset along the intensity axis for clarity. 
Defect Channel Formation between Nanopores

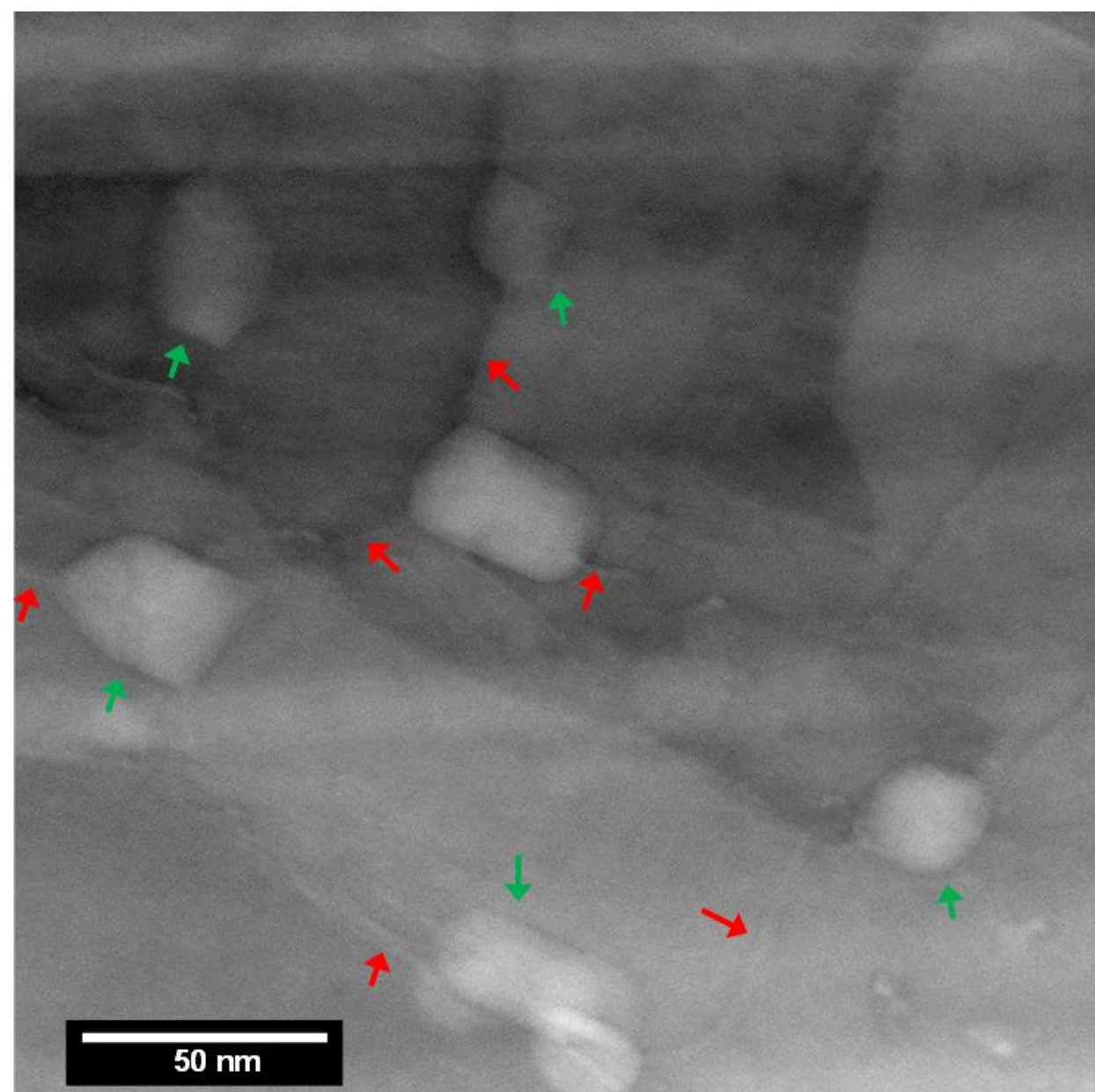

Figure S7. Bright-field STEM image showing pores as bright contrast (denoted by green arrows) and defect channels (denoted by red arrows). 


\section{Intragranular Cracks}

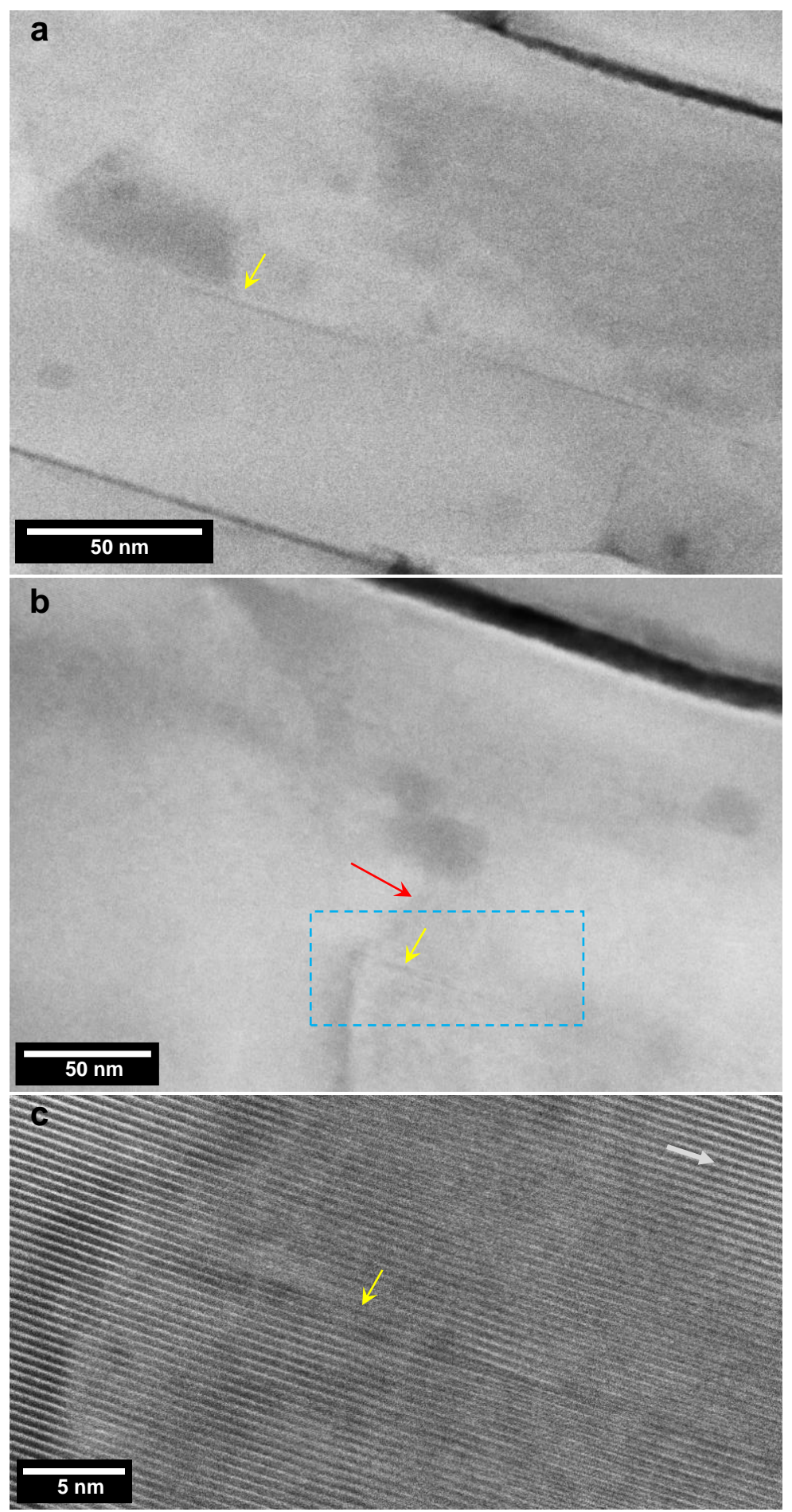

Figure S8. (a) HAADF image showing an intragranular crack through the edge of the nanopore, marked by a yellow arrow, (b) HAADF image showing an intragranular crack initiating from the defect channel, marked by a red arrow, and (c) High-resolution HAADF image from the marked region in 'b' showing crack initiation. 
ToF-SIMS - Time-of-Flight Secondary Ion Mass Spectrometry
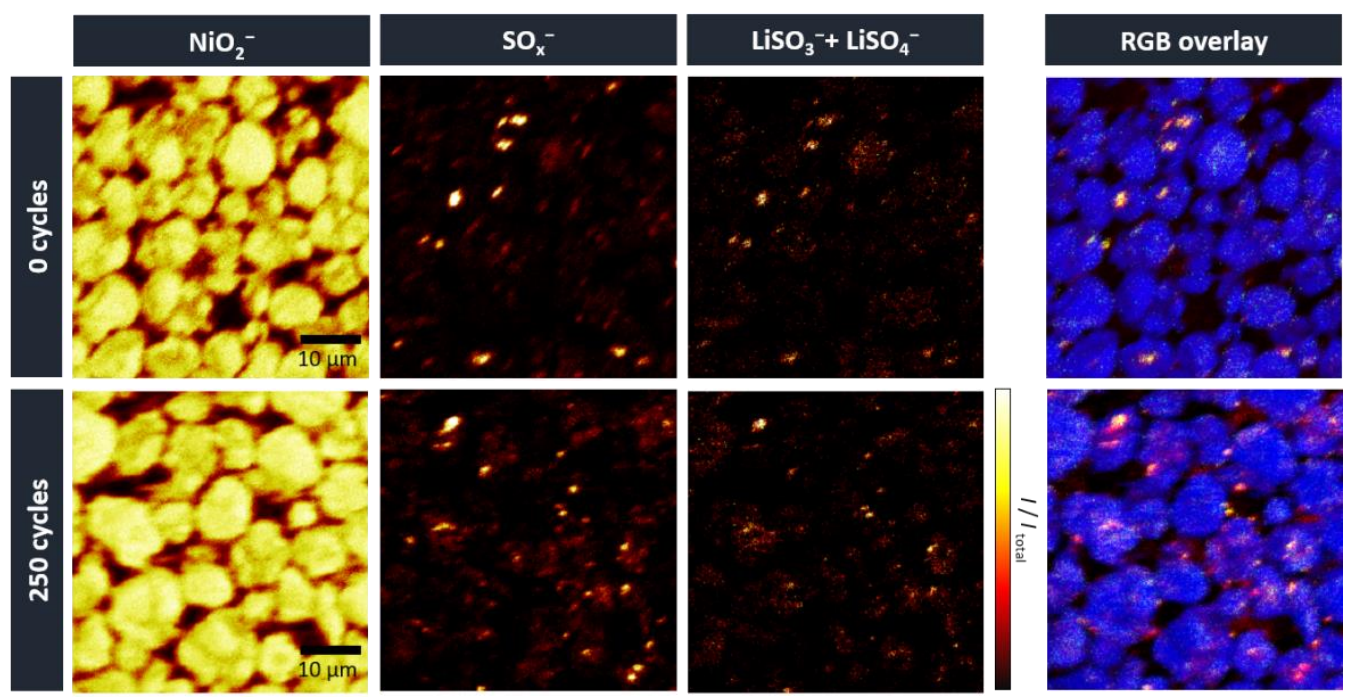

Figure S9. Secondary ion images of exemplary sectional planes within the analyzed volume for both cathodes. The NCM851005 secondary particles are represented by the $\mathrm{NiO}_{2}{ }^{-}$fragment within the $\mathrm{NiO}_{2}{ }^{-}$ mass image on the left. The local enrichments of $\mathrm{SO}_{x}^{-}(0 \leq x \leq 3)$ fragments and the corresponding summated images of $\mathrm{LiSO}_{3}{ }^{-}$and $\mathrm{LiSO}_{4}{ }^{-}$fragments are shown in the middle. On the right, $\mathrm{RGB}$ overlays of the three secondary ion images are shown. It can be seen, that the positions of local enrichments of $\mathrm{SO}_{x}{ }^{-}$ and $\mathrm{LiSO}_{3}{ }^{-}+\mathrm{LiSO}_{4}^{-}$match well.

\section{Primary Grains of LNO}
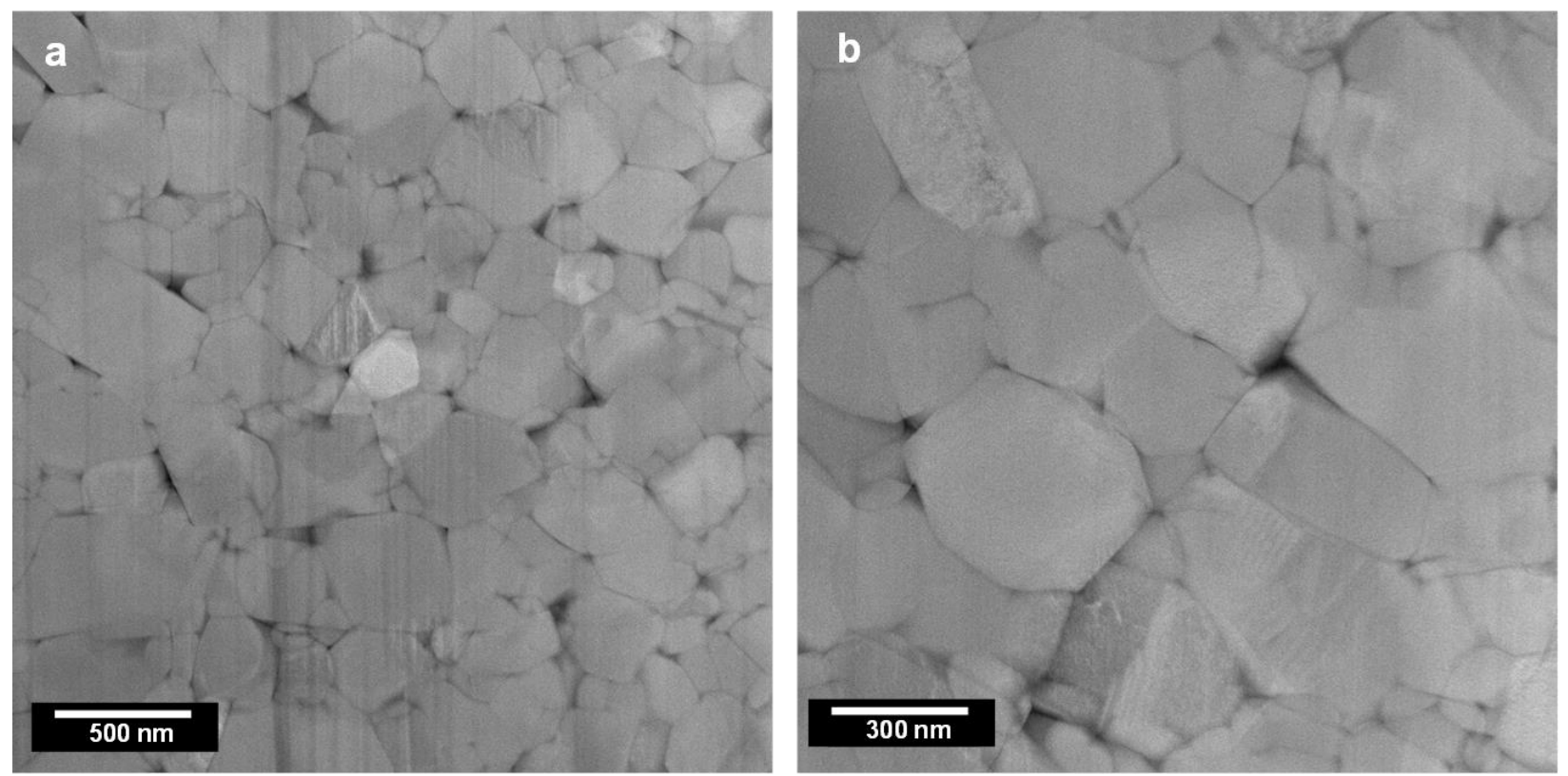

Figure S10. (a, b) Low-magnification images of different regions in LNO showing no intragranular nanopores. 


\section{Sample Preparation (Example of Pristine Material)}
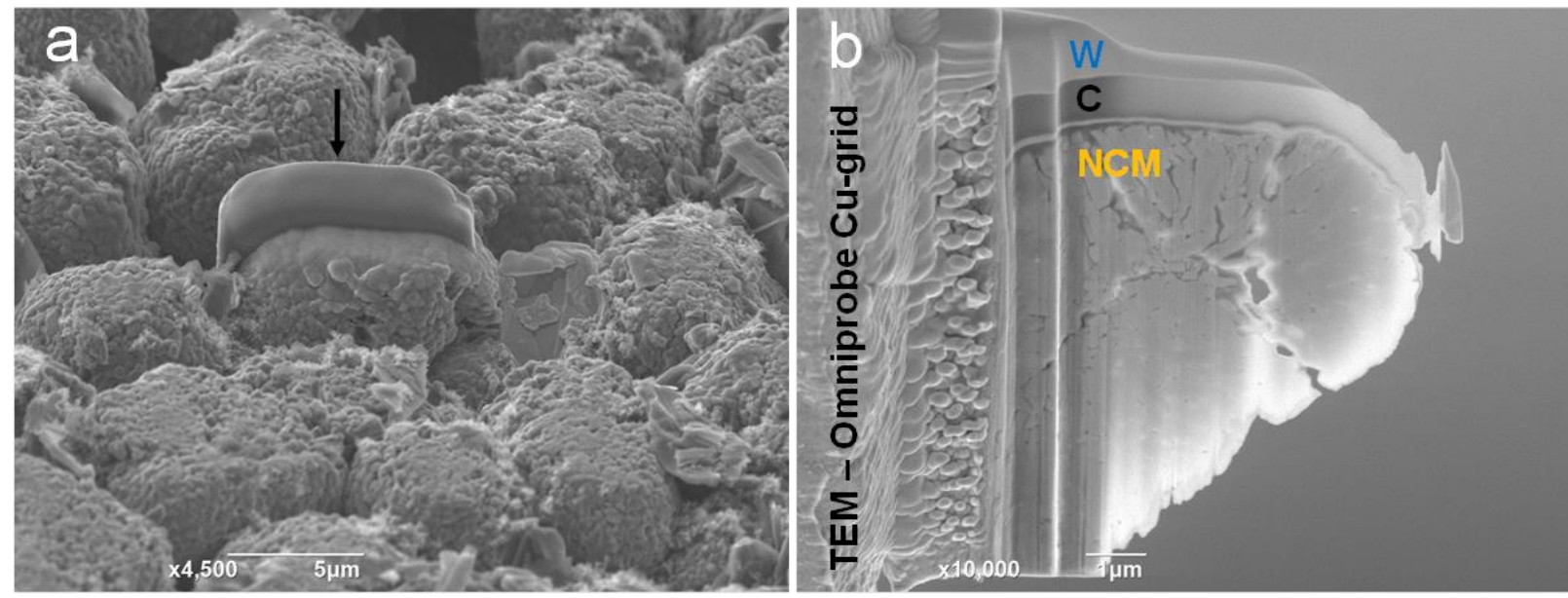

Figure S11. SEM micrographs illustrating FIB sample preparation. (a) Secondary particle selected to prepare the lamellae. The black arrow indicates the protective layers of tungsten $(W)$ and carbon $(C)$. (b) Final electron transparent lamella with strong contrast between the $\mathrm{W}$ layer on top, followed by $\mathrm{C}$ and NCM851005.

\section{Investigation of lon Beam Damage}
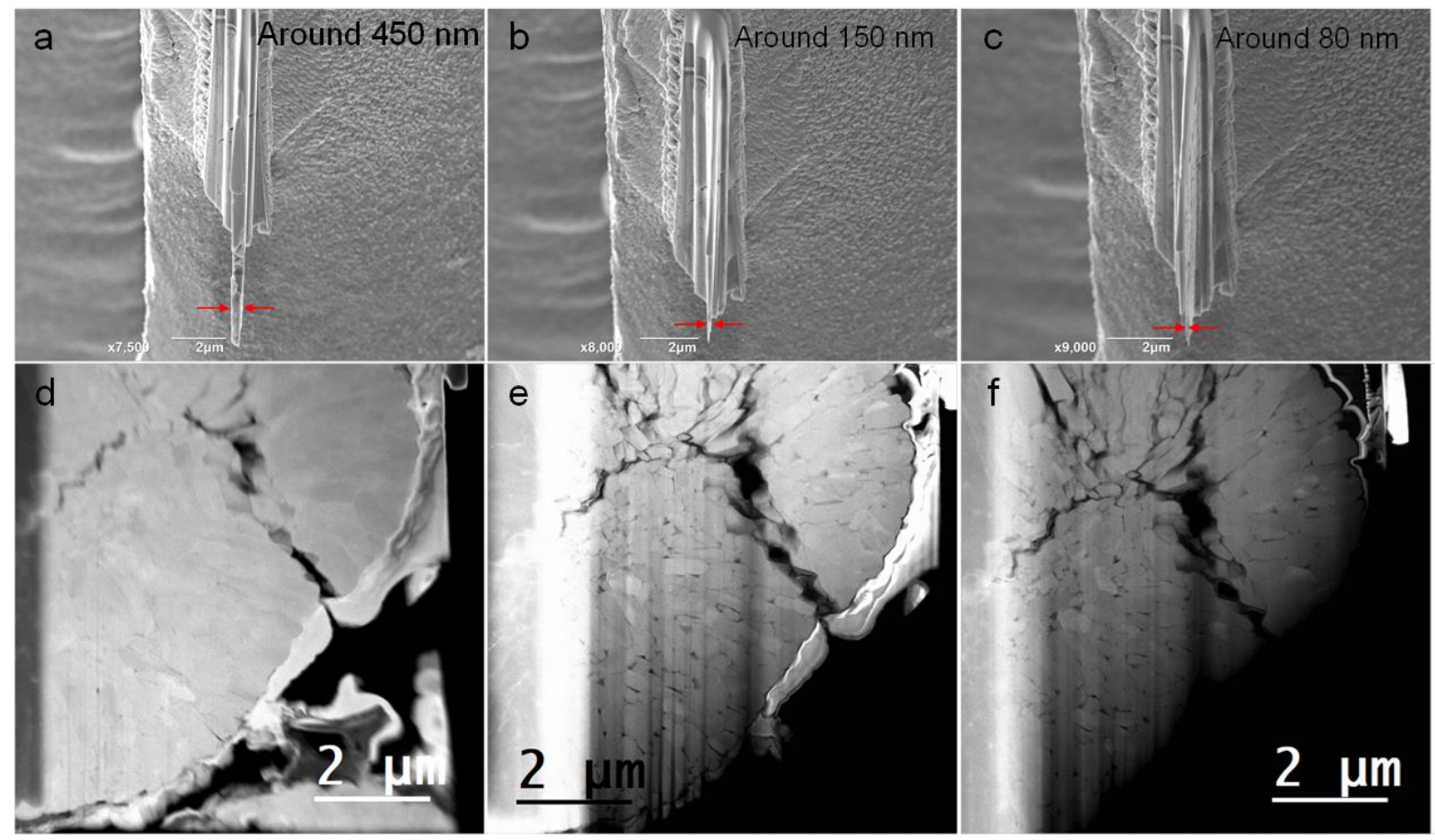

Figure S12.1. SEM micrographs of FIB-prepared lamellae (non-cycled) having different thicknesses (from the region denoted by red arrows) of around $450 \mathrm{~nm}$ (a), $150 \mathrm{~nm}$ (b), and $80 \mathrm{~nm}$ (c), and (d-f) the corresponding STEM images. 


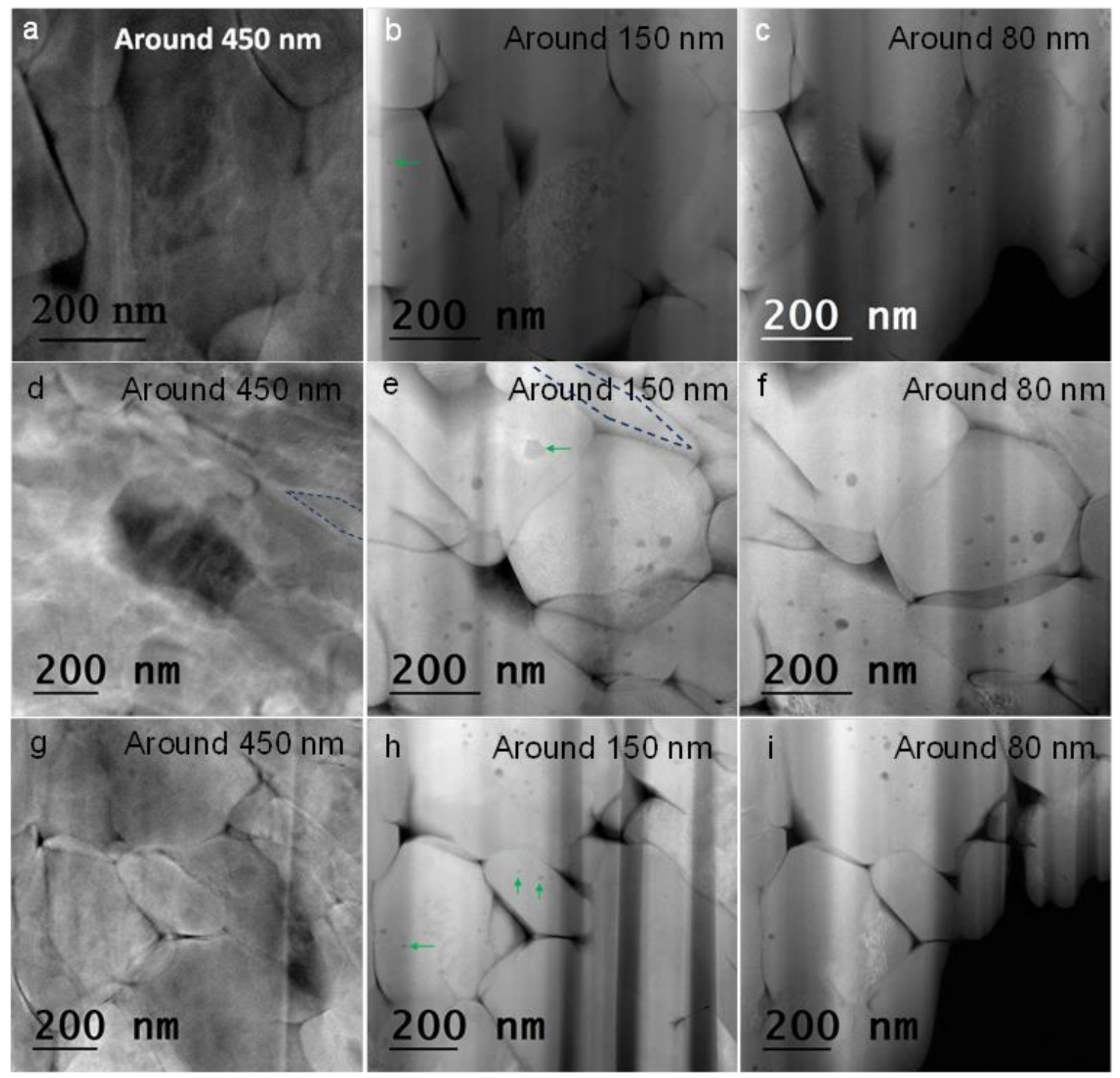

Figure S12.2. Three regions (region 1: $(a-c)$, region 2: (d-f), and region 3: $(g-i)$ ) imaged to investigate any effect of the ion beam on pore formation in non-cycled (pristine) material. No additional pore formation is observed as the sample is being thinned from $450 \mathrm{~nm}$ to $80 \mathrm{~nm}$. In contrast, a reduction in pore number is observed during thinning (denoted by green arrows in (b), (e), and (h) for $150 \mathrm{~nm}$, but absent in (c), (f), and (i) for $80 \mathrm{~nm}$ ) due to the removal of material. The regions in blue in (d) and (e) are used as a marker to recognize the position after each thinning step. 


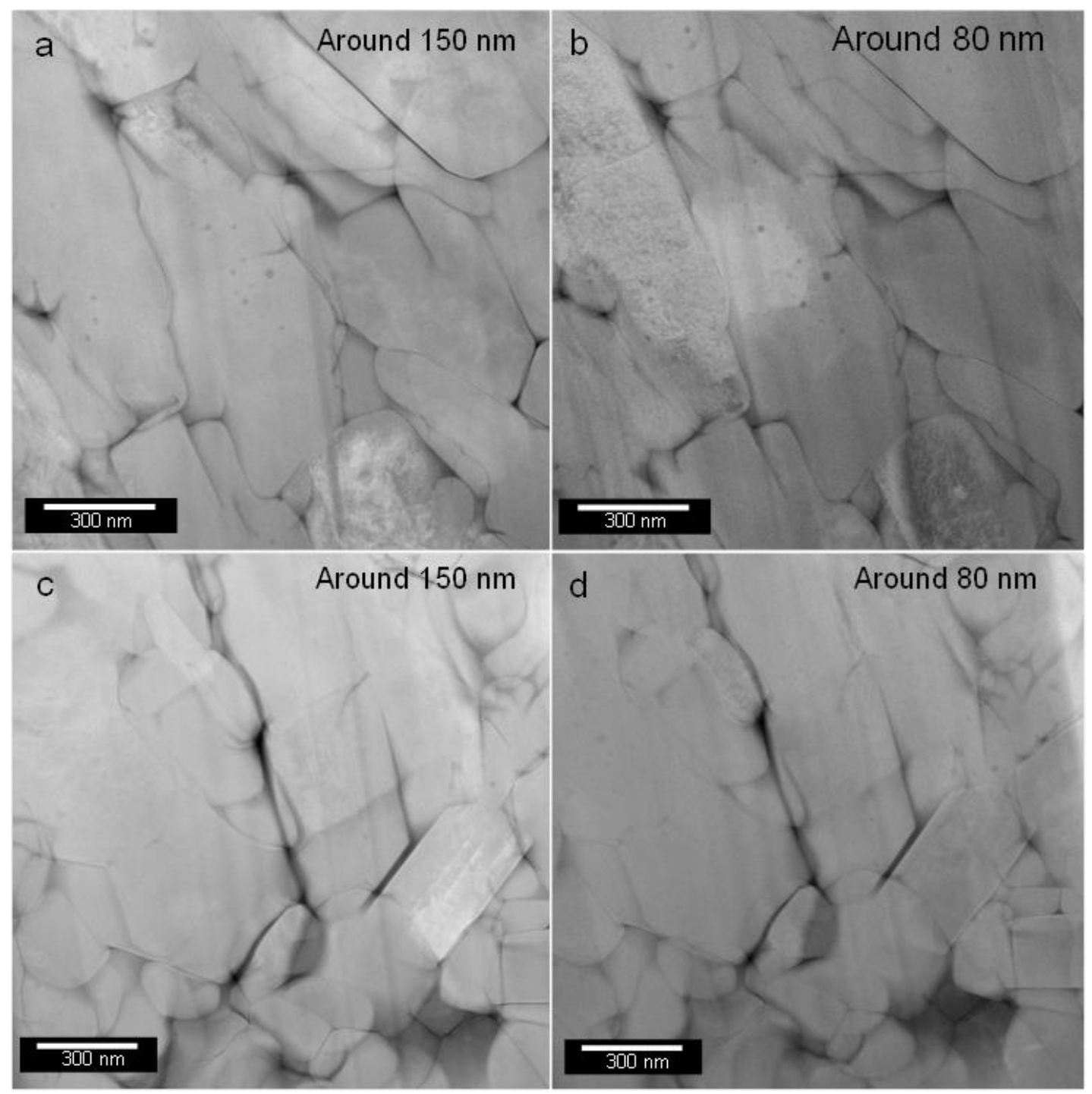

Figure S12.3. Similar experiment performed on a sample after 500 cycles leading to the same conclusion. As is evident (region 1: $(a, b)$ and region 2: $(c, d)$ ), there is no additional pore formation as the lamellae is being thinned from $150 \mathrm{~nm}$ to $80 \mathrm{~nm}$.

\section{References:}

(1) Beyer, A.; Straubinger, R.; Belz, J.; Volz, K. Local Sample Thickness Determination via Scanning Transmission Electron Microscopy Defocus Series. J. Microsc. 2016, Vol. 262, 171-177. 